19

\title{
Оптические свойства сферических металлических наночастиц, покрытых слоем оксида
}

\author{
(C) A.В. Коротун, А.А. Коваль \\ Национальный университет „Запорожская политехника“, \\ 69063 Запорожье, Украина \\ e-mail: andko@zntu.edu.ua
}

Поступила в редакцию 10.04.2019 г.

В окончательной редакции 27.07.2019 г.

Принята к публикации 12.08.2019 г.

В рамках классической теории исследованы оптические свойства ансамбля металлических наночастиц, покрытых слоем оксида. Проанализировано влияние механизмов рассеяния электронов на поляризуемость наночастиц. Рассмотрен предельный случай тонкого оксидного слоя, для которого получены аналитические выражения для действительной и мнимой частей поляризуемости. Исследована эволюция частотных зависимостей поляризуемости и коэффициента экстинкции при вариации размера частиц и толщины оксидного слоя. Показано, что учет размерной зависимости поверхностной составляющей времени релаксации приводит к изменению характера размерной зависимости частоты поверхностных плазмонов двуслойных наночастиц.

Ключевые слова: поляризуемость, металлическая наночастица, оксид, плазмон. DOI: $10.21883 /$ OS.2019.12.48705.133-19

\section{Введение}

Широкое использование металлических наночастиц и проволочек в технике обусловлено преимущественно их уникальными регулируемыми оптическими свойствами, в частности эффективным поглощением света в некотором спектральном диапазоне. Композитные металлдиэлектрические наноструктуры позволяют расширить рабочий диапазон частот по сравнению с металлическими наносистемами. Например, частота плазмонного резонанса в таких структурах может изменяться в более широких пределах [1,2]. Подобная гибкость в регулировании свойств делает композитные наночастицы особенно перспективными для технических применений: при разработке быстродействующих оптических переключателей для систем телекоммуникации, передачи и обработки информации [3,4], оптических наноантенн [5], в медицине [6], катализе [7], при изготовлении датчиков [8], в солнечной энергетике [9-12] и других областях.

Вследствие естественного окисления металла на поверхности наночастицы в жидком или газообразном окружении $[2,13]$ возможно формирование оксидного слоя. Поэтому помимо исследований оптических свойств металлических наночастиц актуальными являются аналогичные исследования для наночастиц металлов с оксидным покрытием [2,14,15].

Кроме того, исследования сферических металлоксидных наночастиц обусловлены уникальной зависимостью их оптических и плазмонных свойств от размера, формы и оптических свойств материалов ядра, оболочки и окружающей диэлектрической среды $[1,2,13-$ 15]. Положение плазмонного резонанса двуслойной наночастицы можно варьировать в широких пределах при изменении размера ядра и толщины оболочки.
Одной из важнейших оптических характеристик наночастиц является их поляризуемость, знание которой, в частности, позволяет рассчитать сечения экстинкции, поглощения и рассеяния.

Существенный интерес также представляет изучение оптических свойств композитов на основе металлоксидных наночастиц. Это обусловлено широким практическим применением таких систем, например в качестве покрытий, изменяющих отражательную способность материалов, сред для оптической записи информации [16] или визуализации клеточных структур [17].

Таким образом, целью работы является исследование частотных зависимостей дипольной поляризуемости и сечения экстинкции сферических двуслойных наночастиц различных металлов, находящихся в различных средах при вариации их радиуса, а также размерной зависимости частоты поверхностных плазмонов с учетом эффекта поверхностного рассеяния электронов в наночастице.

\section{Основные соотношения}

Рассмотрим сферическую металл-оксидную наночастицу радиусом $r$, покрытую слоем оксида толщиной $t$ $(R=r+t$ - полный радиус частицы).

Для малых частиц сечение экстинкции как функцию частоты можно записать в виде [18]

$$
C_{\text {ext }}(\omega)=\frac{4 \pi \omega}{c \sqrt{\epsilon_{m}}} \operatorname{Im} \alpha(\omega),
$$

где $c$ - скорость света, $\alpha-$ поляризуемость частицы, а окружающая среда с проницаемостью $\epsilon_{m}$ считается немагнитной. 
Дипольная поляризуемость $\alpha(\omega)$ наночастицы, покрытой слоем оксида и находящейся в диэлектрике, определяется выражением [19]

$$
\alpha(\omega)=R^{3} \frac{\begin{array}{c}
\left(\epsilon_{\text {oxide }}-\epsilon_{m}\right)\left(2 \epsilon_{\text {oxide }}+\epsilon_{\text {metal }}(\omega)\right)- \\
\left(\epsilon_{\text {oxide }}+2 \epsilon_{m}\right)\left(2 \epsilon_{\text {oxide }}+\epsilon_{\text {oxide }}-\epsilon_{\text {metal }}(\omega)\right) Q^{3} \\
-\left(2 \epsilon_{\text {oxide }}-\epsilon_{m}\right)\left(\epsilon_{\text {oxide }}-\epsilon_{\text {metal }}(\omega)\right) Q^{3}
\end{array}}{}
$$

где $\epsilon_{\text {oxide }}$ - диэлектрическая проницаемость оксида; $Q=1-t / R=1-q$.

Подставляя в выражение (2) комплекснозначную диэлектрическую функцию металла, получаем следующее выражение:

$$
\alpha(\omega)=R^{3} \frac{\operatorname{Re} \Lambda+i \operatorname{Im} \Lambda}{\Xi},
$$

где введены обозначения

$$
\begin{aligned}
\Xi \equiv & {\left[\left(\epsilon_{\text {oxide }}+2 \epsilon_{m}\right)\left(2 \epsilon_{\text {oxide }}+\epsilon_{1}\right)\right.} \\
& \left.-2\left(\epsilon_{\text {oxide }}-\epsilon_{m}\right)\left(\epsilon_{\text {oxide }}-\epsilon_{1}\right) Q^{3}\right]^{2} \\
& +\epsilon_{2}^{2}\left[\epsilon_{\text {oxide }}+2 \epsilon_{m}+2\left(\epsilon_{\text {oxide }}-\epsilon_{m}\right) Q^{3}\right]^{2} ;
\end{aligned}
$$

$\operatorname{Re} \Lambda \equiv\left(\epsilon_{\text {oxide }}-\epsilon_{m}\right)\left(\epsilon_{\text {oxide }}+2 \epsilon_{m}\right)\left[\left(\epsilon_{1}+2 \epsilon_{\text {oxide }}\right)^{2}+\epsilon_{2}^{2}\right]$

$+Q^{3}\left[\left(\epsilon_{1}-\epsilon_{\text {oxide }}\right)\left(\epsilon_{1}+2 \epsilon_{\text {oxide }}\right)\left(4 \epsilon_{\text {oxide }}^{2}+4 \epsilon_{m}^{2}+3 \epsilon_{\text {oxide }} \epsilon_{m}\right)\right.$

$\left.+2 \epsilon_{2}^{2}\left(2 \epsilon_{\text {oxode }}^{2}+3 \epsilon_{m}^{2}+\epsilon_{\text {oxide }} \epsilon_{m}\right)\right]+2 Q^{6}\left(\epsilon_{\text {oxide }}-\epsilon_{m}\right)$

$\times\left[\left(2 \epsilon_{\text {oxide }}+\epsilon_{m}\right)\left(\epsilon_{1}-\epsilon_{\text {oxide }}\right)^{2}+2 \epsilon_{2}^{2}\left(\epsilon_{\text {oxide }}+\epsilon_{m}\right)\right] ;$

$$
\operatorname{Im} \Lambda=27 \epsilon_{m} \epsilon_{\text {oxide }}^{2} \epsilon_{2} Q^{3},
$$

а $\epsilon_{1}$ и $\epsilon_{2}$ представляют собой соответственно действительную и мнимую части диэлектрической функции металла.

Рассмотрим предельные случаи:

1) Тонкий слой оксида $(q \ll 1)$. При этом, учитывая, что в первом порядке по малому параметру $q$ $Q^{3} \cong 1-3 q$ и $Q^{6} \cong 1-6 q$, и подставляя эти соотношения в выражения (4)-(6), получаем в том же самом приближении (см. Приложение А):

$$
\begin{aligned}
& \operatorname{Re} \alpha(\omega)=\frac{3\left(3 \epsilon_{\text {oxide }}+\epsilon_{m}\right) \epsilon_{2}^{2}+9 \epsilon_{1}^{2} \epsilon_{\text {oxide }}-18 \epsilon_{\text {oxide }} \epsilon_{m}^{2}-}{-4 \epsilon_{\text {oxide }}^{2} \epsilon_{m}+2 \epsilon_{m} \epsilon_{1}^{2}+11 \epsilon_{1} \epsilon_{\text {oxide }} \epsilon_{m}} \\
& \times\left\{1-q\left[4 \frac{\left(\epsilon_{\text {oxide }}-\epsilon_{m}\right)\left(\left(\left(\epsilon_{1}+2 \epsilon_{m}\right)\left(\epsilon_{\text {oxide }}-\epsilon_{1}\right)-\epsilon_{2}^{2}\right)\right)}{\epsilon_{\text {oxide }}\left(\epsilon_{\text {oxide }}^{2}+\left(\epsilon_{1}+2 \epsilon_{m}\right)^{2}\right)}\right.\right. \\
& \left.\left.+\frac{2 \epsilon_{2}^{2}\left(6 \epsilon_{\text {oxide }}+\epsilon_{m}-\left(\epsilon_{m}^{2} / \epsilon_{\text {oxide }}\right)-16 \epsilon_{\text {oxide }} \epsilon_{m}^{2}+12 \epsilon_{1} \epsilon_{\text {oxide }}\left(\epsilon_{1}-\epsilon_{\text {oxide }}\right)-\right.}{-\epsilon_{m}\left(2 \epsilon_{\text {oxide }}^{2}+\epsilon_{1}^{2}\right)+11 \epsilon_{1} \epsilon_{\text {oxide }} \epsilon_{m}+12 \epsilon_{m}^{2} \epsilon_{1}}\right]\right\}, \\
& \operatorname{Im} \alpha(\omega)=R^{3} \frac{3 \epsilon_{m} \epsilon_{2}}{\epsilon_{\text {oxide }}^{2}+\left(\epsilon_{1}+2 \epsilon_{m}\right)^{2}} \\
& \times\left\{1-q\left[3-4 \frac{\left(\epsilon_{\text {oxide }}-\epsilon_{m}\right)\left(\left(\epsilon_{1}+2 \epsilon_{m}\right)\left(\epsilon_{1}-\epsilon_{\text {oxide }}\right)+\epsilon_{2}^{2}\right)}{\epsilon_{\text {oxide }}\left(\epsilon_{\text {oxide }}^{2}+\left(\epsilon_{1}+2 \epsilon_{m}\right)^{2}\right)}\right]\right\} .
\end{aligned}
$$

2) Толстый слой оксида $(q \rightarrow 1, Q \rightarrow 0)$. В этом случае для действительной и мнимой частей поляризуемости получаются следующие выражения:

$$
\begin{gathered}
\operatorname{Re} \alpha(\omega)=\frac{\epsilon_{\text {oxide }}-\epsilon_{m}}{\epsilon_{\text {oxide }}+2 \epsilon_{m}}, \\
\operatorname{Im} \alpha(\omega)=R^{3} \frac{27 \epsilon_{m} \epsilon_{\text {oxide }}^{2} \epsilon_{2} Q^{3}}{\left(\epsilon_{\text {oxide }}+2 \epsilon_{m}\right)\left[\left(\epsilon_{1}+2 \epsilon_{\text {oxide }}\right)^{2}+\epsilon_{2}^{2}\right]} .
\end{gathered}
$$

Формулы (9), (10) свидетельствуют о том, что с уменьшением содержания металлической фракции действительная часть поляризуемости стремится к постоянной величине, а мнимая к нулю. Полученный результат соответствует случаю диэлектрической среды, содержащей сферические включения другого диэлектрика.

Для диэлектрической функции металлической наночастицы воспользуемся выражением Друде

$$
\epsilon_{\text {metal }}(\omega)=\epsilon_{1}(\omega)+i \epsilon_{2}(\omega)=\epsilon^{\infty}-\frac{\omega_{p}^{2} \tau^{2}}{1+\omega^{2} \tau^{2}}+i \frac{\omega_{p}^{2} \tau}{\omega\left(1+\omega^{2} \tau^{2}\right)}
$$

Здесь $\epsilon^{\infty}$ компонента, описывающая вклад ионного остова; $\omega_{p}^{2}=e^{2} n_{e} / \epsilon_{0} m^{*}-$ плазменная частота, $e$ и $n_{e}$ - заряд и концентрация электронов соответственно $\left(n_{e}=3 / 4 \pi r_{s}^{3}, r_{s}\right.$ - среднее расстояние между электронами), $\epsilon_{0}$ - диэлектрическая постоянная вакуума, а $m^{*}$ - эффективная масса электронов, $\tau$ - время релаксации.

Плазмонный резонанс в малых металлических наночастицах, покрытых слоем оксида, возникает при условии [20]

$$
\operatorname{Re} \epsilon_{\text {metal }}\left(\omega_{s p}\right)=-2 \epsilon_{\text {oxide }} \frac{\epsilon_{\text {oxide }} q+\epsilon_{m}(3-q)}{\epsilon_{\text {oxide }}(3-2 q)+2 \epsilon_{m} q} .
$$

Если $q \rightarrow 1$, то условие резонанса определяется выражением $\operatorname{Re} \epsilon_{\text {metal }}\left(\omega_{s p}\right)=-2 \epsilon_{\text {oxide }}$ (ядро „воспринимает“ оксид как окружающую его среду), тогда как для $q=0$ (случай непокрытых частиц) условие резонанса принимает „традиционный“ вид $\operatorname{Re}\left(\omega_{s p}\right)=-2 \epsilon_{m}$. В общем случае выражение для частоты поверхностных плазмонов можно получить, используя соотношения (11) и (12),

$$
\omega_{s p}=\sqrt{\frac{\omega_{p}^{2}}{\epsilon^{\infty}+2 \epsilon_{\text {oxide }} \frac{\epsilon_{\text {oxide }} q+\epsilon_{m}(3-q)}{\epsilon_{\text {oxide }}(3-2 q)+2 \epsilon_{m} q}}-\frac{1}{\tau^{2}}} .
$$

В предельных случаях имеем:

1) для тонкого слоя оксида

$$
\begin{aligned}
\operatorname{Re} \epsilon_{\text {metal }}\left(\omega_{s p}\right) & =-2 \epsilon_{\text {oxide }} \frac{3 \epsilon_{m}+q\left(\epsilon_{\text {oxide }}-\epsilon_{n}\right)}{3 \epsilon_{\text {oxide }}+2 q\left(\epsilon_{m}-\epsilon_{\text {oxide }}\right)} \\
& \cong-2 \epsilon_{m}\left[1+\frac{q}{3}\left(1+\frac{\epsilon_{\text {oxide }}}{\epsilon_{m}}-\frac{2 \epsilon_{m}}{\epsilon_{\text {oxide }}}\right)\right],
\end{aligned}
$$

откуда

$$
\omega_{s p}=\sqrt{\frac{\omega_{p}^{2}}{\epsilon^{\infty}+2 \epsilon_{m}\left[1+\frac{q}{3}\left(1+\frac{\epsilon_{\text {oxide }}}{\epsilon_{m}}-\frac{2 \epsilon_{m}}{\epsilon_{\text {oxide }}}\right)\right]}-\frac{1}{\tau^{2}}},
$$


Таблица 1. Параметры металлов

\begin{tabular}{c|c|c|c}
\hline \multirow{2}{*}{$r_{s} / a_{0}[23]$} & $\mathrm{Al}$ & $\mathrm{Cu}$ & $\mathrm{Ag}$ \\
\cline { 2 - 4 } & 2.07 & 2.11 & 3.02 \\
\hline$\epsilon^{\infty}$ & 1 & $12.03[24]$ & $3.7[24]$ \\
\hline$m^{*} / m_{e}$ & $1.48[25]$ & $1.49[26]$ & $0.96[26]$ \\
\hline$\tau, \mathrm{fs}[23]$ & 8 & 27 & 40
\end{tabular}

Таблица 2. Параметры оксидов и матрицы

\begin{tabular}{c|c|c|c}
\hline \multicolumn{2}{c|}{ Оксид } & \multicolumn{2}{c}{ Матрица } \\
\hline \multicolumn{2}{c|}{$\epsilon_{\text {oxide }}$} & \multicolumn{2}{c}{$\epsilon_{m}$} \\
\hline $\mathrm{Al}_{2} \mathrm{O}_{3}$ & $\mathrm{Cu}_{2} \mathrm{O}$ & $\mathrm{KCl}$ & тефлон \\
\hline $3.13[27]$ & $3.7[28]$ & $2.1[29]$ & $2.3[30]$
\end{tabular}

или

$$
\begin{aligned}
& \omega_{s p} \cong \sqrt{\frac{\omega_{p}^{2}}{\epsilon^{\infty}+2 \epsilon_{m}}-\frac{1}{\tau^{2}}} \\
& \times\left[1-\frac{\epsilon_{m} \omega_{p}^{2}}{3\left(\epsilon^{\infty}+2 \epsilon_{m}\right)\left(\frac{\omega_{p}^{2}}{\epsilon^{\infty}+2 \epsilon_{m}}-\frac{1}{\tau^{2}}\right)}\left(1+\frac{\epsilon_{\text {oxide }}}{\epsilon_{m}}-\frac{2 \epsilon_{n}}{\epsilon_{\text {oxide }}}\right) q\right]
\end{aligned}
$$

2) для толстого слоя оксида

$$
\omega_{s p}=\sqrt{\frac{\omega_{p}^{2}}{\epsilon^{\infty}+2 \epsilon_{\text {oxide }}}-\frac{1}{\tau^{2}}} ;
$$

3) в отсутствие оксида $(q=0)$

$$
\omega_{s p}=\sqrt{\frac{\omega_{p}^{2}}{\epsilon^{\infty}+2 \epsilon_{m}}-\frac{1}{\tau^{2}}} .
$$

Отметим, что время релаксации $\tau$ можно считать постоянным только для объемных металлов. В случае, когда какой-либо из геометрических размеров металлических структур меньше длины свободного пробега электронов в 3D-металле, то кроме рассеяния электронов на фононах и примесях необходимо также учитывать рассеяние на границах нанообъекта. Поэтому в формулу (11) для диэлектрической функции вместо $\tau$ входит его эффективное значение $\tau_{\mathrm{ef}}$, которое определяют как [21]:

$$
\frac{1}{\tau_{\mathrm{ef}}}=\frac{1}{\tau}+A(\omega, r) \frac{v_{\mathrm{F}}}{r} .
$$

где $A(\omega, r)$ - эффективный параметр, описывающий степень потери когерентности при рассеянии электрона на поверхности, который в общем случае зависит от радиуса наночастицы и частоты падающего излучения; $v_{\mathrm{F}}$ - скорость Ферми.
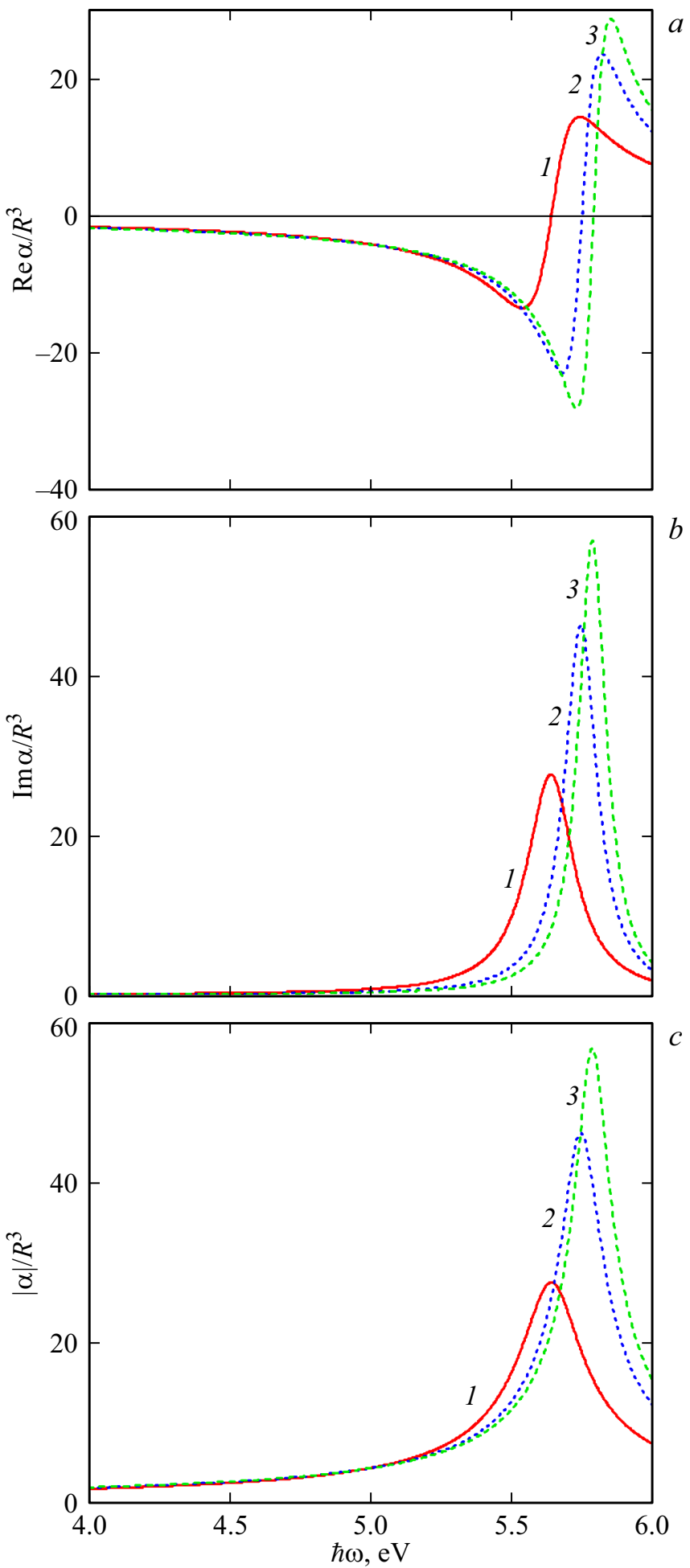

Рис. 1. Частотные зависимости действительной $(a)$ и мнимой $(b)$ частей поляризуемости и ее модуля $(c)$ для наночастиц $\mathrm{Al} @ \mathrm{Al}_{2} \mathrm{O}_{3}$ в $\mathrm{KCl}$ разного радиуса: $1-r=10 \mathrm{~nm} ; 2-$ $r=20 \mathrm{~nm} ; 3-r=30 \mathrm{~nm}$.

Вклад второго слагаемого в (17) тем больше, чем меньше размеры металлических кластеров. Так, для частиц с радиусом $r \simeq 10-100 \mathrm{~nm}$ второе слагаемое может 

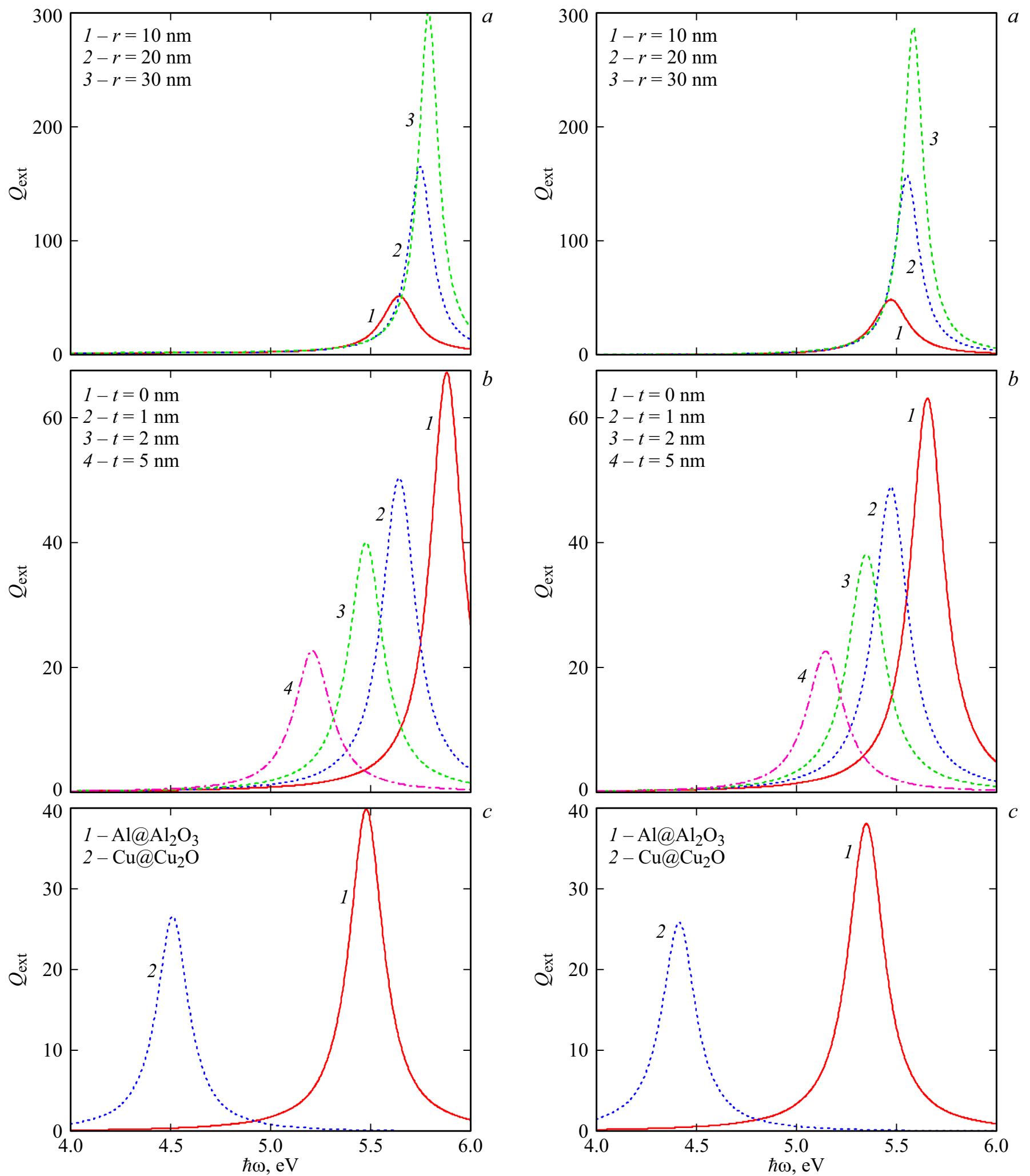

Рис. 2. Частотные зависимости коэффициента экстинкции для наночастиц $\mathrm{Al} @ \mathrm{Al}_{2} \mathrm{O}_{3}$ разного радиуса $(a)$ и с различной толщиной оксидного покрытия $(b)$ и наночастиц различных металлов $(c)$ в $\mathrm{KCl}$.

Рис. 3. Частотные зависимости коэффициента экстинкции для наночастиц $\mathrm{Al} @ \mathrm{Al}_{2} \mathrm{O}_{3}$ разного радиуса $(a)$ и с различной толщиной оксидного покрытия $(b)$ и наночастиц различных металлов $(c)$ в тефлоне. 


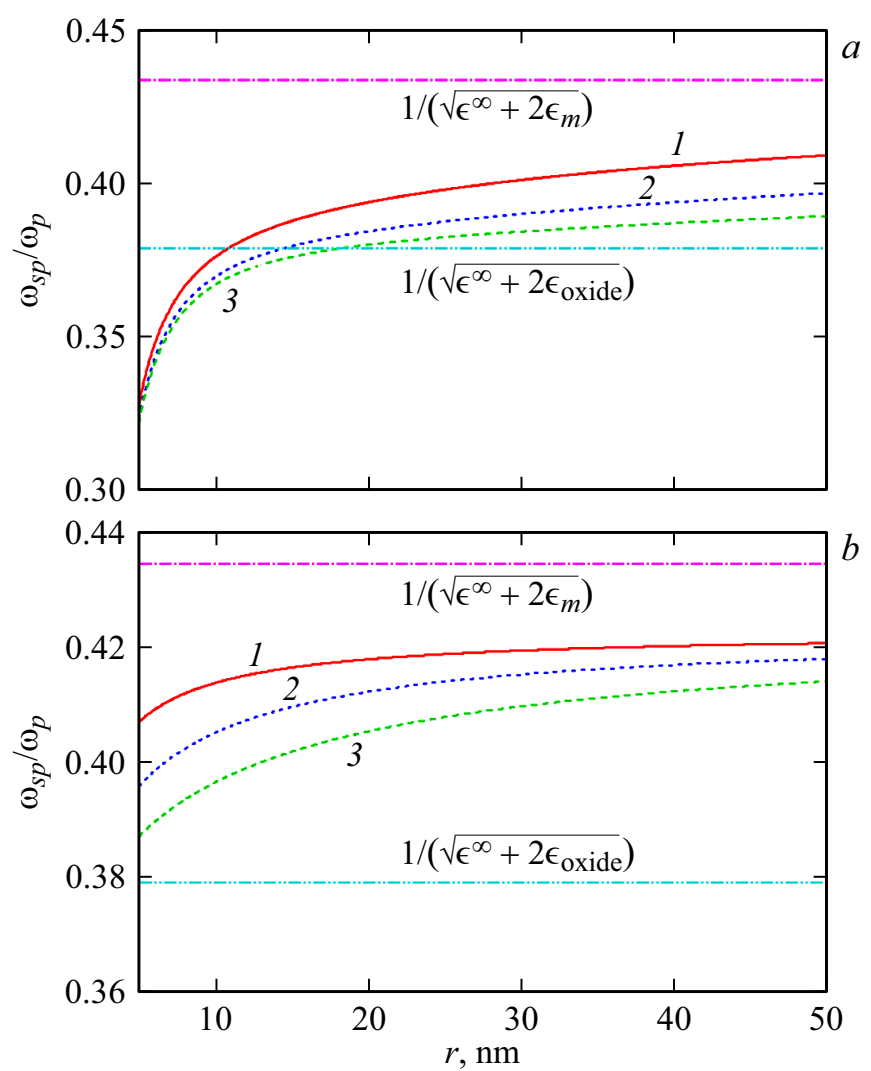

Рис. 4. Размерные зависимости частоты поверхностных плазмонов для наночастиц $\mathrm{Al} @ \mathrm{Al}_{2} \mathrm{O}_{3}$ с различной толщиной оксидного покрытия в тефлоне: $1-t=2 \mathrm{~nm} ; 2-t=5 \mathrm{~nm}$; $3-t=10 \mathrm{~nm}$ при различных подходах к определению $A: a-$ $A=\frac{3}{4} ; b-A=A(\omega, r)$.

стать существенно больше первого, и выражение (17) при этом принимает вид

$$
\frac{1}{\tau_{\mathrm{eff}}} \cong A(\omega, r) \frac{v_{\mathrm{F}}}{r}
$$

Кинетический подход, примененный в работах [22] при описании процессов рассеяния на поверхности и в объеме сферических металлических наночастиц, позволил получить следующее выражение для параметра $A(\omega, r)$ :

$$
A(\omega, r)=\frac{1}{4}\left(\frac{\omega_{p}}{\omega}\right)^{2}\left[1-\frac{2 v_{s}}{\omega} \sin \frac{\omega}{v_{s}}+\frac{2 v_{s}^{2}}{\omega^{2}}\left(1-\cos \frac{\omega}{v_{s}}\right)\right],
$$

где $v_{s}=v_{\mathrm{F}} / 2 r$.

Отметим, что для наночастиц в вакууме $\left(\epsilon_{m}=1\right)$, когда $\omega \rightarrow \omega_{p} / \sqrt{1+2 \epsilon_{m}}$ и $v_{s} \ll \omega$, (19) приводит к известному результату $A=3 / 4$, и тогда можно записать

$$
\frac{1}{\tau_{\mathrm{ef}}}=\frac{1}{\tau}+\frac{3}{4} \frac{v_{F}}{r}
$$

Выражения (17), (19) и (20) используются в дальнейшем для расчета размерной зависимости частоты поверхностных плазмонов.

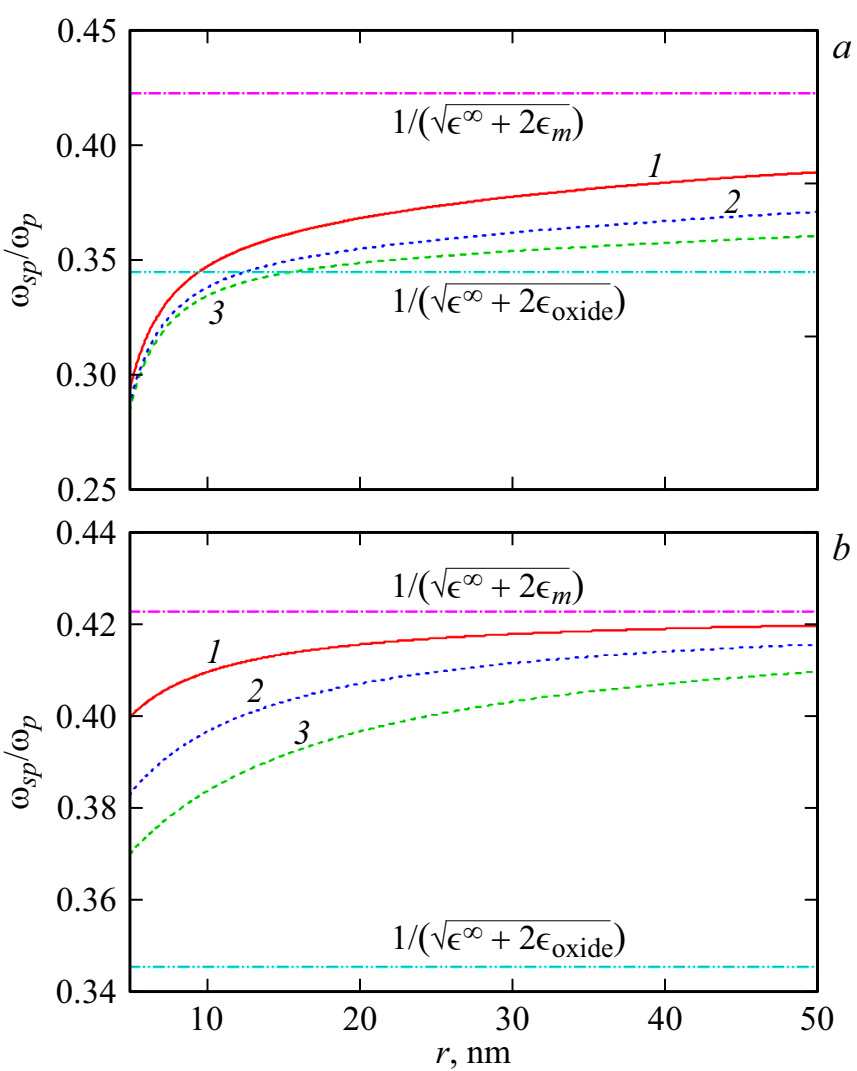

Рис. 5. Размерные зависимости частоты поверхностных плазмонов для наночастиц $\mathrm{Cu} @ \mathrm{Cu}_{2} \mathrm{O}$ с различной толщиной оксидного покрытия в тефлоне: $1-t=2 \mathrm{~nm} ; 2-t=5 \mathrm{~nm}$; $3-t=10 \mathrm{~nm}$ при различных подходах к определению $A: a-$ $A=\frac{3}{4} ; b-A=A(\omega, r)$.

\section{Результаты вычислений и их обсуждение}

Вычисления проводились для композитов на основе металл-оксидных наночастиц алюминия, меди и серебра различных радиусов, с разной толщиной оксидного слоя, находящихся в различных матрицах. Параметры металлов, оксидов и диэлектриков приведены в табл. 1 и 2 соответственно.

На рис. 1 представлены графики частотных зависимостей действительной и мнимой частей поляризуемости и ее модуля для наночастиц $\mathrm{Al}$ разного радиуса, покрытых слоем оксида толщиной $1 \mathrm{~nm}$. Следует отметить, что функция $\operatorname{Re} \alpha(\omega)$ (рис. $1, a)$ в отличие от $\operatorname{Im} \alpha(\omega)$ (рис. $1, b)$ является знакопеременной и имеет близко расположенные экстремумы при $\hbar \omega_{0} \approx 5.5 \mathrm{eV}$. Причем чем больше размер частицы, тем больше $|\operatorname{Re} \alpha(\omega)|$ и $\operatorname{Im} \alpha(\omega)$. Кроме того, с увеличением размера наночастиц происходит сдвиг экстремумов $\operatorname{Re} \alpha(\omega)$ и $\operatorname{Im} \alpha(\omega)$ в область больших частот, что свидетельствует о возможности управления оптическими свойствами путем варьирования размеров наночастиц. Частотная зависимость модуля поляризуемости (рис. 1,c) практически во всем частотном интервале качественно подобна зависимости 
$\operatorname{Im} \alpha(\omega)$, поскольку $\operatorname{Re} \alpha(\omega)$ сравнима по величине с $\operatorname{Im} \alpha(\omega)$ только в области $\omega \approx \omega_{0}$.

Графики частотных зависимостей коэффициента экстинкции $Q_{\text {ext }} \quad\left(Q_{\text {ext }}=C_{\text {ext }} / \pi R^{2}\right)$ для наночастиц $\mathrm{Al} @ \mathrm{Al}_{2} \mathrm{O}_{3}$ приведены на рис. 2, a, b. Указанные кривые имеют один ярко выраженный пик при $\hbar \omega_{0} \approx 5.5-6.0 \mathrm{eV}$ для наночастиц разного радиуса и толщиной оксидного слоя $t=1 \mathrm{~nm}$ (рис. 2,a). Причем с увеличением относительной толщины $q=t / R$ оксидного слоя значение $Q_{\text {ext }}\left(\omega_{0}\right)$ уменьшается (рис. $\left.2, b\right)$, а сами максимумы сдвигаются в область меньших частот, что связано с уменьшением доли металлической фракции в общем объеме металл-оксидной частицы. Этой же причиной объясняется „красный“ сдвиг максимума нормированного сечения экстинкции для наночастиц Al радиусом $r=10 \mathrm{~nm} \mathrm{с} \mathrm{увеличением} \mathrm{толщины} \mathrm{оксидной} \mathrm{оболоч-}$ ки. Зависимости $Q_{\operatorname{ext}(\omega)}$ для наночастиц $\mathrm{Al} @ \mathrm{Al}_{2} \mathrm{O}_{3}$ и $\mathrm{Cu} @ \mathrm{Cu}_{2} \mathrm{O}$ одинакового радиуса и одинаковой толщины оксидного слоя $(r=10 \mathrm{~nm}, t=2 \mathrm{~nm})$ приведены на рис. 2,c. В этом случае положение максимумов и значения $Q_{\text {ext }}\left(\omega_{0}\right)$ определяются концентрацией свободных электронов.

Для сравнения на рис. 3 приведены аналогичные результаты для частиц $\mathrm{Al} @ \mathrm{Al}_{2} \mathrm{O}_{3}$ и $\mathrm{Cu} @ \mathrm{Cu}_{2} \mathrm{O}$ в тефлоне. Как можно заметить, качественно результаты подобны, а количественно отличаются незначительно. Это свидетельствует о том, что на оптические свойства композита в рассматриваемом спектральном диапазоне определяющее влияние имеют размеры частиц и толщина оксидного слоя, а не тип матрицы.

Кривые размерной зависимости частоты поверхностных плазмонов для случая наночастиц $\mathrm{Al}$ и $\mathrm{Cu}$, покрытых слоем оксида различной толщины, изображены на рис. 4 и 5. Отметим, что в случае $A=3 / 4$ (рис. 4, $a$ и 5,a) функция $\omega_{s p} / \omega_{p}=f(r)$ возрастает и при $r \rightarrow \infty$ стремится к своем асимптотическому значению $1 / \sqrt{\epsilon^{\infty}+2 \epsilon_{m}}$, а при увеличении толщины оксидного слоя дисперсионные кривые „прижимаются“ горизонтальной прямой $1 / \sqrt{\epsilon^{\infty}+2 \epsilon_{\text {oxide }}}$. В случае, когда эффективный параметр $A$ зависит от радиуса металлоксидной наночастицы (рис. $4, b$ и $5, b$ ), поведение размерных зависимостей частоты поверхностных плазмонов практически не зависит от материала композитной наночастицы. Для наночастиц $\mathrm{Al} @ \mathrm{Al}_{2} \mathrm{O}_{3}$ и $\mathrm{Cu} @ \mathrm{Cu}_{2} \mathrm{O}$ указанные кривые асимптотически приближаются к горизонтальной прямой $1 / \sqrt{\epsilon^{\infty}+2 \epsilon_{m}}$, причем в случае наночастиц $\mathrm{Cu} @ \mathrm{Cu}_{2} \mathrm{O}$ они располагаются ближе к этой прямой. Это связано с тем, что значение $1 / \sqrt{\epsilon^{\infty}+2 \epsilon_{m}}$ для $\mathrm{Cu} @ \mathrm{Cu}_{2} \mathrm{O}$ меньше, чем для $\mathrm{Al} @ \mathrm{Al}_{2} \mathrm{O}_{3}$. Следует отметить, что незначительная разница значений для $\omega_{s p} / \omega_{p}$ композитных наночастиц $\mathrm{Al} @ \mathrm{Al}_{2} \mathrm{O}_{3}$ и $\mathrm{Cu} @ \mathrm{Cu}_{2} \mathrm{O}$ связана с компенсацией влияния величин $\epsilon^{\infty}$ и $A(r)$ для этих объектов. Кроме того, чем меньше толщина оксидного слоя для наночастиц $\mathrm{Al} @ \mathrm{Al}_{2} \mathrm{O}_{3}$ и $\mathrm{Cu} @ \mathrm{Cu}_{2} \mathrm{O}$, тем выше расположены дисперсионные кривые.

\section{Заключение}

Получены частотные зависимости коэффициента экстинкции, действительной, мнимой частей и модуля поляризуемости, а также размерные зависимости частоты поверхностных плазмонов для сферических металлоксидных наночастиц. Рассмотрены предельные случаи тонкой и толстой оксидной оболочек.

Установлена возможность управления изменениями оптических свойств металл-оксидных наночастиц путем изменения их размеров.

Показано, что максимальное значение коэффициента экстинкции увеличивается при увеличении толщины оксидного слоя, а максимумы сдвигаются в область больших частот, что обусловлено уменьшением доли металлической фракции в общем объеме металл-оксидной частицы. Кроме того, численные значения коэффициента экстинкции в данном частотном диапазоне определяются преимущественно радиусом частиц и толщиной оксидной оболочки и практически не зависят от материала матрицы.

Продемонстрировано, что частота поверхностных плазмонов растет с увеличением размера наночастиц, а расположение кривых $\omega_{s p} / \omega_{p}=f(r)$ зависит от характера размерной зависимости эффективного параметра, описывающего степень потери когерентности, и слабо зависит от материала частиц-включений.

\section{Конфликт интересов}

Авторы заявляют, что у них нет конфликта интересов.

\section{Приложение А. Формулы для действительной и мнимой частей поляризуемости}

Получим выражения для $\Xi^{-1}$ и $\Lambda$ в первом порядке по малому параметру $q$ :

$$
\begin{aligned}
\Xi & =\left[2 \epsilon_{\text {oxide }}^{2}+\epsilon_{\text {oxide }} \epsilon_{1}+2 \epsilon_{1} \epsilon_{m}+4 \epsilon_{\text {oxide }} \epsilon_{m}\right. \\
& -\left(2 \epsilon_{\text {oxide }}^{2}+2 \epsilon_{1} \epsilon_{m}+2 \epsilon_{1} \epsilon_{\text {oxide }}-2 \epsilon_{\text {oxide }} \epsilon_{m}\right. \\
& \times(1-3 q)]^{2}+\epsilon_{2}^{2}\left[\epsilon_{\text {oxide }}+2 \epsilon_{m}+2\left(\epsilon_{\text {oxide }}-\epsilon_{m}\right)(1-3 q)\right]^{2} \\
& =9\left\{\left[\epsilon_{\text {oxide }}\left(\epsilon_{1}+2 \epsilon_{m}\right)+2 q\left(\epsilon_{\text {oxide }}-\epsilon_{m}\right)\left(\epsilon_{\text {oxide }}-\epsilon_{1}\right)\right]^{2}\right. \\
& \left.+\epsilon_{2}^{2}\left[\epsilon_{\text {oxide }}-2 q\left(\epsilon_{\text {oxide }}-\epsilon_{m}\right)\right]^{2}\right\} \\
& =9 \epsilon_{\text {oxide }}^{2}\left(\epsilon_{\text {oxide }}^{2}+\left(\epsilon_{1}+2 \epsilon_{m}\right)^{2}\right) \\
& \times\left\{1+4 q \frac{\left(\epsilon_{\text {oxide }}-\epsilon_{m}\right)\left(\epsilon_{2}^{2}+\left(\epsilon_{1}+2 \epsilon_{m}\right)\left(\epsilon_{\text {oxide }}-\epsilon_{1}\right)\right)-\epsilon_{2}^{2}}{\epsilon_{\text {oxide }}\left(\epsilon_{\text {oxide }}^{2}+\left(\epsilon_{1}+2 \epsilon_{m}\right)^{2}\right)}\right\},
\end{aligned}
$$


Тогда

$$
\begin{aligned}
& \Xi^{-1}=\frac{1}{9 \epsilon_{\text {oxide }}^{2}\left(\epsilon_{2}^{2}+\left(\epsilon_{1}+2 \epsilon_{m}\right)^{2}\right)} \\
& \times\left\{1-4 q \frac{\left(\epsilon_{\text {oxide }}-\epsilon_{m}\right)\left[\left(\epsilon_{1}+2 \epsilon_{m}\right)\left(\epsilon_{\text {oxide }}-\epsilon_{1}\right)-\epsilon_{2}^{2}\right]}{\epsilon_{\text {oxide }}\left(\epsilon_{2}^{2}+\left(\epsilon_{1}+2 \epsilon_{m}\right)^{2}\right)}\right\}
\end{aligned}
$$

Аналогично

$$
\left.\begin{array}{l}
\operatorname{Re} \Lambda=3 \epsilon_{\text {oxide }}\left(3 \epsilon_{\text {oxide }}+\epsilon_{m}\right) \epsilon_{2}^{2}+9 \epsilon_{\text {oxide }}^{2} \epsilon_{1}^{2}-18 \epsilon_{\text {oxide }}^{2} \epsilon_{m}^{2} \\
-4 \epsilon_{\text {oxide }}^{3} \epsilon_{m}+2 \epsilon_{\text {oxide }} \epsilon_{m} \epsilon_{1}^{2}+11 \epsilon_{\text {oxide }}^{2} \epsilon_{m} \epsilon_{1}-3 q \\
\times\left[2 \epsilon_{2}^{2}\left(+\epsilon_{\text {oxide }}^{2}-\epsilon_{m}^{2}+\epsilon_{\text {oxide }} \epsilon_{m}\right)-16 \epsilon_{\text {oxide }}^{2} \epsilon_{m}^{2}+12 \epsilon_{\text {oxide }}^{2} \epsilon_{1}^{2}\right. \\
-12 \epsilon_{\text {oxide }}^{3} \epsilon_{1}-2 \epsilon_{\text {oxide }}^{3} \epsilon_{m}-\epsilon_{\text {oxide }} \epsilon_{m} \epsilon_{1}^{2}+11 \epsilon_{\text {oxide }}^{2} \epsilon_{m} \epsilon_{1} \\
\left.+12 \epsilon_{\text {oxide }} \epsilon_{m}^{2} \epsilon_{1}\right]=\left(3 \epsilon_{\text {oxide }}\left(3 \epsilon_{\text {oxide }}+\epsilon_{m}\right) \epsilon_{2}^{2}+9 \epsilon_{\text {oxide }}^{2} \epsilon_{1}^{2}\right. \\
\left.-18 \epsilon_{\text {oxide }}^{2} \epsilon_{m}^{2}-4 \epsilon_{\text {oxide }} \epsilon_{m}+2 \epsilon_{\text {oxide }} \epsilon_{m} \epsilon_{1}^{2}+11 \epsilon_{\text {oxiode }}^{2} \epsilon_{m} \epsilon_{1}\right) \\
\times\left\{1-3 q\left[2 \epsilon_{2}^{2}\left(6 \epsilon_{\text {oxide }}^{2}-\epsilon_{m}^{2}+\epsilon_{\text {oxide }} \epsilon_{m}\right)-16 \epsilon_{\text {oxide }}^{2} \epsilon_{m}^{2}\right.\right. \\
+12 \epsilon_{\text {oxide }}^{3} \epsilon_{1}-2 \epsilon_{\text {oxide }}^{3} \epsilon_{m}-\epsilon_{\text {oxide }} \epsilon_{m} \epsilon_{1}^{2}+11 \epsilon_{\text {oxide }}^{2} \epsilon_{m} \epsilon_{1} \\
\left.+12 \epsilon_{\text {oxide }} \epsilon_{m}^{2} \epsilon_{1}\right]\left[3 \epsilon_{\text {oxide }}\left(3 \epsilon_{\text {oxide }}+\epsilon_{m}\right) \epsilon_{2}^{2}+9 \epsilon_{\text {oxide }}^{2} \epsilon_{1}^{2}\right. \\
\left.-18 \epsilon_{\text {oxide }}^{2} \epsilon_{m}^{2}-4 \epsilon_{\text {oxide }}^{3} \epsilon_{m}+2 \epsilon_{\text {oxide }} \epsilon_{m} \epsilon_{1}^{2}+11 \epsilon_{\text {oxide }}^{2} \epsilon_{m} \epsilon_{1}\right]
\end{array}\right\} ;
$$

Подставляя (A1)-(А3) в (3), получаем для действительной и мнимой частей поляризуемости формулы (7), (8).

\section{Список литературы}

[1] Kulish V.V., Tomchuk P.M. // Surf. Sci. 2008. V. 602. N 5. P. 1045.

doi 10.1016/j.susc.2007.12.030

[2] Santillán J.M.J., Scaffardi L.B., Schinca D.C. // J. Phys. D: Appl. Phys. 2011. V. 44. N 10. id. 105104. doi 10.1088/0022-3727/44/10/105104

[3] Chakraborty P. // J. Mater. Sci. 1998. V. 33. N 9. P. 2235. doi 10.1023/A:1004306501659

[4] Xia H.-Y., Teng Ch.-X., Zhao X.-W., Zheng J. // Chin. Phys. Lett. 2012. V. 29. N 8. id. 084215. doi 10.1088/0256-307X/29/8/084215

[5] Zuev D.A., Makarov S.V., Mukhin I.S., Starikov S.V., Morozov I.A., Shishkin I.I., Krasnok A.E., Belov P.A. // eprint arXiv:1601.02013. 2016

[6] Астафьева Л.Г., Залесская Г.А., Плавский В.Ю. // Опт. и спектр. 2012. Т. 112. N 4. С. 697; Astafyeva L.G., Zalesskaya G.A., Plavskii V.Yu. // // Opt. Spectrosc. 2012. V. 112. N 4. P. 642. doi 10.1134/S0030400X12040030

[7] Narayanan R., Tabor C., El-Sayed M.A. // Top. Catal. 2008. V. 48. P. 60
[8] D'Agata R., Palladino P., Spoto G. // Beilstein J. Nanotechnol. 2017. V. 8. P. 1. doi:10.3762/bjnano.8.1

[9] Liu Z., Lee S.Y., Lee E.-Ch. // Appl. Phys. Lett. 2014. V. 105. N 22. id.223306. doi 10.1063/1.4903749

[10] Pustovalov V.K., Astafyeva L.G., Fritzsche W. // Sol. Ener. 2015. V. 122. P. 1334. doi

[11] Liu Ch., Zhang D., Liu Y., Wu D., Chen L., Ma R., Yu Z., Yu L., Ye H. // Nanosc. Res. Lett. 2017. V. 12. N 1. id.601. doi 10.1186/s11671-017-2363-7

[12] Mlinar V. // Nanotechnology. 2013. V. 24. N 4. id. 042001. doi 10.1088/0957-4484/24/4/042001

[13] Santillán J.M.J., Videla F.A., Fernández van Raap M.B., Schinca D.C., Scaffardi L.B. // J. Appl. Phys. 2013. V. 113. $\mathrm{N}$ 13. id. 134305. doi $10.1063 / 1.4798387$

[14] Астафьева Л.Г., Пустовалов В.К. // Опт. и спектр. 2016. T. 121. N 1. C. 118; Astafyeva L.G., Pustovalov V.K. // Opt. Spectrosc. 2016. V. 121. N 1. P. 109. doi $10.1134 / \mathrm{S} 0030400 \mathrm{X} 16070043$

[15] Астафьева Л.Г., Пустовалов В.К., Фритче В. // Опт. и спектр. 2019. Т. 126. № 3. С. 374; Astafyeva L.G., Pustovalov V.K., Fritzsche W. // Opt. Spectrosc. 2019. V. 126. N 3.

[16] Qu S., Du C., Song Y., Wang Y., Gao Y., Liu S., Li Y., Zhu D. // Chem. Phys. Lett. 2002. V. 356. P. 403. doi 10.1016/S0009-2614(02)00396-2

[17] Rosi N.L., Mirkin C.A. // Chem. Rev. 2005. V. 105. P. 1547. doi 10.1021/cr030067f

[18] Monreal R.C, Apell S.P, Antosiewicz T.J. // Opt. Expr. 2014. V. 22(21). P. 24994. doi 10.1364/OE.22.024994

[19] Bohren C.F., Huffman D.R. Absorption and Scattering of Light by Small Particles. John Wiley \& Sons, 2008. 530 p.

[20] Tapia V.R., Franco A., Macedo J.G. // J. Nanopart. Res. 2012. V. 14. P. 915. doi 10.1007/s11051-012-0915-4

[21] Kreibig U., Vollmer M. Optical properties of metal clusters. Berlin: Springer, 1995. $532 \mathrm{p}$.

[22] Grigorchuk N.I., Tomchuk P.M. // Phys. Rev. B. 2011. V. 84. N 8. id. 085448 . doi 10.1103/PhysRevB.84.085448

[23] Ашкрофт Н., Мермин Н. Физика твердого тела. Т. 1. М.: Мир, 1979. 400 c.

[24] Shaganov I.I., Perova T.S., Berwick K. // Photon. Nanostruct. - Fundam. and Applicat. 2017. V. 27. P. 24.

[25] Харрисон У. Теория твердого тела. М.: Мир, 1972. 616 с.

[26] Johnson P.B., Christy R.W. // Phys. Rev. B. 1972. V. 6. P. 4370. doi 10.1103/PhysRevB.6.4370

[27] Zhu J., Li J.-J., Zhao J.-W. // Appl. Surf. Sci. 2014. V. 314. P. 145. doi 10.1016/j.apsusc.2014.06.129

[28] Ching W.Y., Xu Y.-N. // Phys. Rev. B. 1989. V. 40. P. 7684. doi 10.1103/PhysRevB.40.7684

[29] Cummings K.D., Garland J.C., Tanner D.B. // Phys. Rev. B. 1984. V. 30. P. 4170. doi 10.1103/PhysRevB.30.4170

[30] Воронкова E.M. и др. Оптические материалы для инфракрасной техники: Справочное издание. М.: Наука, 1965. 335 c. 